\title{
Community Health Workers Engagement in Prevention of Mother-to-Child Transmission of HIV (PMTCT): A Cross-Sectional Study from Selected Fishing Communities in Tanzania
}

\author{
Maseke Richard Mgabo ${ }^{1,2, *}$, Sia Msuya ${ }^{1,3}$, Declare Mushi ${ }^{1}$ \\ ${ }^{1}$ Institute of Public Health, Kilimanjaro Christian Medical University College, Moshi, Tanzania \\ ${ }^{2}$ Department of Population Studies, Institute of Rural Development Planning, Dodoma, Tanzania \\ ${ }^{3}$ Kilimanjaro Christian Medical Centre, Moshi, Tanzania
}

Email address:

mmgabo@irdp.ac.tz (M. R. Mgabo)

${ }^{*}$ Corresponding author

\section{To cite this article:}

Maseke Richard Mgabo, Sia Msuya, Declare Mushi. Community Health Workers Engagement in Prevention of Mother-to-Child Transmission of HIV (PMTCT): A Cross-Sectional Study from Selected Fishing Communities in Tanzania. Journal of Family Medicine and Health Care. Vol. 6, No. 2, 2020, pp. 39-45. doi: 10.11648/j.jfmhc.20200602.12

Received: March 31, 2019; Accepted: May 23, 2019; Published: May 28, 2020

\begin{abstract}
The prevalence of HIV infection is reported to be higher in fishing communities of Lake Victoria compared to non fishing neighboring communities. Besides, access to HIV/AIDS related services has been reported to be either inadequate or unavailable to some of these fishing communities particularly for those residing in islands. This study explored the engagement of Community Health Workers (CHWs) in provision of PMTCT services among the fishing communities of Sengerema and Musoma District Councils (DCs) in the Lake Zone of Tanzania. This was a cross sectional study using qualitative approach. Intrinsic and extrinsic motives were found to influence community members to be engaged as CHWs. The majority of CHWs were involved in Antenatal Care (ANC) and Reproductive and Child Health (RCH) as well as in health promotion activities as a strategy to cope with the shortage of trained health staff. CHWs were found to assist in HIV/AIDS Counseling and Treatment Center (CTC), generally, CHWs were not directly engaged in provision of PMTCT services. On the other hand, the National Health Policy does not clearly set roles of the CHWs and their incentives. Nevertheless, there are potentials of people willingness to volunteer in community health works that need to be better leveraged. Deploying CHWs is critical to enabling accessible primary health care services including HIV/AIDS particularly PMTCT services in remote areas such as fishing communities where human resource for health is reported to be inadequate.
\end{abstract}

Keywords: Community Health Workers, Fishing Communities, Lake Victoria, HIV/AIDS, Prevention of Mother-to-Child Transmission of HIV, Sengerema, Musoma, Tanzania

\section{Background}

Evidences have shown that CHWs have been important key players in provision of primary health care services in resource limited settings as well as in remote regions $[1,2]$. Globally, CHWs have differently named but literally referring to members of a specific community that are selected by, and answerable to the community where they work, supported by the local health system, and receiving less training than formally trained health workers [3].
However, CHWs vary in terms of their recruitment, training, supervision, type and amount of work, and form of remuneration both within and across countries, [4, 5]. Previous studies have recommended that if CHWs are well integrated into the health care system, they might have a profound effect on achievement on Sustainable Development Goal 3 [6] "Ensuring a healthy life and promoting well-being for all ages" a goal which is most transversal goals and interconnected with the other SDGs. The potentials of CHWs for ensuring healthy life and promoting well being for all 
ages is evidenced from South Africa [7], Rwanda [5, 8], Kenya [9], Malawi [10, 11] and Tanzania [12, 13]. All of these studies reported on the engagement of CHWs in bridging the gap caused by shortage of Human Resources for Health (HRH); at the same time, enabling essential health services to reach the isolated and hard to reach communities.

Fishing communities are categorized as hard-to-reach communities $[11,14]$. They are also considered to be most at risk population $[15,16]$. Still health services in fishing communities are reported to be inadequate [15-17]. Shortage of $\mathrm{HRH}$ is one of the factors impeding health services provision, including HIV/AIDS related services [18, 19] in these communities. Furthermore, unavailability of HIV/AIDS services subjects fishing communities to HIV susceptibility than their neighboring non-fishing communities $[17,18]$ and women are reported to be more affected than men (Kiwanuka et al. 2014, Mafigiri et al. 2017, Opio et al. 2013). Elsewhere, CHWs have been used to curb the gap of HRH in hard to reach communities in rural areas [2, 7, 21, 22]. Limited information is available regarding the engagement of CHWs in enhancing the implementation of PMTCT programs in fishing communities of Lake Victoria on the Tanzania's side. This study presents findings on the engagement of CHWs in the implementation of PMTCT programs in selected fishing communities of Lake Victoria in Musoma Rural and Sengerema DCs.

\section{Methods}

\subsection{Study Design and Area}

This was a cross-sectional study which employed qualitative approach. The study was conducted in Musoma and Sengerema DCs located in northern Tanzania, in Mara and Mwanza Regions respectively. Some parts of these DCs are situated along the shores of Lake Victoria. During the time of this study, Sengerema DC had an estimated population of 663,034, (Male 330,018, Female 333,016) of which 143,592 were women of reproductive age [23]. The DC had 50 health facilities; one Designated District Hospital (owned by the Roman Catholic Church), 4 health centers and 45 dispensaries (44 owned by the government) (Ag DMO, Sengerema District Council, Pers, comm. 2016). Musoma DC was estimated to have a population of 178,356 people (Male 87,324, Female 91,032), an estimate of women of reproductive age being 38,631 [23]. At the time of this study, Musoma DC was reported to have only one government health center and 26 dispensaries, of which 23 were government owned and 3 privately owned (Ag DMO, Musoma District Council, Pers, Comm 2016)

HIV prevalence among people aged 15 - 49 years was estimated to be 5\% for both Musoma and Sengerema [24] which is almost equal to the $5.1 \%$ national HIV prevalence [25]. Fishing is the most affluent economic activities though done at small-scale level. Apart from fishing, other economic activities found in these DCs include; small scale trades, small scale gold mining, agriculture and livestock keeping
$[26,27]$. These DCs were selected based on their involvement in fishing activities [28] and their HIV prevalence [29] coupled with inadequate HRH as well as unavailability or inadequate HIV/AIDS health related services [30]. This study was conducted from October 2015 to February 2016. This was a period towards and after the target of reach zero infants HIV infection target by 2015 [31].

\subsection{Study Population, Sample Size and Sampling Procedure}

The study population included Key Informants (KIs) who were health care providers and CHW's from Musoma and Sengerema DCs. The inclusion criteria were health care providers or CHWs who have worked for at least one year or more in either ANC/PMTCT unit or Counseling and Treatment Center (CTC) in the study area and consented in writing.

The current study used a purposive sampling to get the respondents who had the characteristics relevant to the study objective. In total, 25 participants were reached after saturation point.

Table 1. List of Key Informants by their cadre.

\begin{tabular}{llll}
\hline Cadre & Sengerema & Musoma & Total \\
\hline Assistant Nurses & 4 & 3 & 7 \\
Nurse Midwife & 3 & 2 & 5 \\
Clinical Officer & 2 & 2 & 4 \\
Community Health Workers & 6 & 3 & 9 \\
Total & 15 & 10 & 25 \\
\hline
\end{tabular}

The KIs involved in this study included 16 health care providers (12 female, 4 males), and 9 CHW's ( 3 males, 6 female). The sample size reached as considered to be adequate compared to previous studies conducted on a similar theme $[32,33]$. KIs were recruited from the health facilities found within a range of 1 to 5 kilometers along the shores of Lake Victoria or in the Islands. These facilities were within the fishing communities' domains, being in a walking distance of less than one hour. It was therefore assumed that majority of the clients to these facilities would be from fishing communities.

\subsection{Data Collection Tools}

\subsubsection{Interview Guide}

The KI interview guide was developed in English then translated and interviewed in Kiswahili, which is a national and common language used by the majority of participants in this study. To explore on the study objective, the interview guide focused on the factors influencing individuals to become CHWs, the perceived CHWs working environment and factors compelling individuals to continue or drop from being CHWs.

\subsubsection{Facilitation of the Key Informants Interview}

Due to its exploratory nature, the current study consists only of qualitative data, whereby one-to-one interview was conducted. The main researcher and two trained research assistants did the data collection. The main researcher led the interviews as per interview guide so as to keep the interview 
on track and consistency. The assistant researchers recorded the conversations. A typical opening question was "In your opinion, what could be the factors pushing a person to become a community health worker?" Next the Key Informants were asked to give their experiences regarding CHWs working environment (in both private/ NGOs and government settings). Then the interview centered on factors compelling individuals to be engaged as CHWs, common interventions involved the CHWs, advantages and challenges as well as their involvement in implementing PMTCT interventions.

Twenty five (25) interviews were conducted and audio taped using digital voice recorder and later transcribed into verbatim (typed in word format). Field notes were completed within 24 hours of the interviews to protect data integrity. All key informants consented to be recorded. All interviews were conducted at health facilities, in rooms with privacy and interview time ranged between 60 to 90 minutes. Data saturation was reached after 17 interviews. However, researchers proceeded and in total 25 interviews were conducted.

\subsection{Data Analysis}

Audio recorded information were analyzed using a thematic analysis approach [34]. The analysis was carried out in three stages beginning with systematic reading of the field notes (familiarization), followed by line-by-line coding and labeling of relevant words and phrases. Codes were then categorized into descriptive and analytical themes. This step took place independently by the main researcher (RM). Then all researchers determined final coding by analyzing and interpreting underlying concepts within participants' responses and isolating key themes. Seventeen (17) codes were generated. Codes were further interpreted and categorized into eleven (11) descriptive sub-codes. These sub-codes were further categorized into five (5) analytical themes (Table 2). After evaluating final themes for consistency, researchers determined the headings used to describe results in this paper.

Table 2. Analytical frame work: CHWs involvement in PMTCT intervention.

\begin{tabular}{|c|c|c|c|}
\hline Codes & Descriptive themes & Analytical themes & Overarching theme \\
\hline $\begin{array}{l}\text { Pushing factors } \\
\text { Economic gains } \\
\text { Social status } \\
\text { Obligations } \\
\text { Religious obligation } \\
\text { Community Role } \\
\text { Sympathy } \\
\text { A strategy to reach many } \\
\text { people } \\
\text { Interested to work with NGO } \\
\text { Common interventions with } \\
\text { CHWs } \\
\text { Achievements } \\
\text { Constraints /challenges } \\
\text { Pressure from family } \\
\text { Meeting basic needs } \\
\text { Time consuming } \\
\text { Volunteering does not pay } \\
\text { Variations between } \\
\text { Government and NGO CHWs } \\
\text { nurturing }\end{array}$ & $\begin{array}{l}\text { Some get motivated with grains or cash. } \\
\text { Some felt respected by saving other life. } \\
\text { Some loved to become doctors/ nurses but their dreams were } \\
\text { not met, so being a CHW is compensating their wishes } \\
\text { Being a CHW some feel obliged to work from religious } \\
\text { perspectives } \\
\text { Some were selected by village authority } \\
\text { Just sympathizing with the people in need } \\
\text { Some are PLWHA so the help colleagues and have been } \\
\text { approached by GOVT or NGOs for purpose } \\
\text { Government rarely provide motivation; NGOs provide working } \\
\text { gears (Bicycle, rain coats, gumboots, umbrella, T-shirts } \\
\text { MNCH intervention such as immunization, nutrition, oral } \\
\text { vaccination, Vitamin A supplementation } \\
\text { Awareness creation and sensitization }\end{array}$ & $\begin{array}{l}\text { Government vs. NGO } \\
\text { Nurturing, working } \\
\text { environment and maintaining } \\
\text { CHWs } \\
\text { Common interventions } \\
\text { undertaken by CHWs } \\
\text { Achievements }\end{array}$ & $\begin{array}{l}\text { Working as CHW in } \\
\text { PMTCT intervention } \\
\text { Working as CHW in } \\
\text { PMTCT intervention }\end{array}$ \\
\hline
\end{tabular}

\section{Results}

Across the interviews, various motivations were identified to motivate individuals who became CHW's. These included; compensating for unrealized ambitions; fulfillment of moral or community, obligations (religious or community), influence by local health personnel, and economic gains. The presentation of the current study results is guided by the analytical themes identified (Table 2).

The following are some of the direct quotes as given by respondents during interviews;

\subsection{Compensating for Unrealized Ambitions}

"I have been here in the village as a fisherman cum a farmer. I wished to be a Clinician but I missed the opportunity because I did not manage to reach even secondary education level. So when Plan International came with a Reproductive and Child Health Project, I picked interest to work with them. Fortunate enough, they involved me as a CHW" (CHW, Male aged 32 from Sengerema DC).

"My secondary school results were not good enough to allow me to proceed with advanced level studies. I had the passion of being a nurse!" Thanks God I'm a CHW (CHW, female aged 28 from Musoma DC)

\subsection{Fulfillment of Moral or Community Obligations}

"I am a sympathetic person, during the early days of HIV/AIDS; I was nursing my sister who suffered from HIV/AIDS. Many families stigmatized their affected 
relatives, some families used to ask me to nurse their patients in return of little money or grains. I become famous! ...then the village authority proposed me to work with the dispensary. That is how I became a CHW and here I am moving on". (Male CHW aged 56 from Sengerema DC).

"I see my Christianity in helping the sick and those in need indeed...this is what our Lord Jesus ordered us to do! This is what pushed me into volunteering as a CHW" (CHW man, aged 40 from Sengerema DC).

\subsection{Influences from Health Personnel}

"She [a Nurse] was alone working at ANC, she was my closest friend and I sympathized with the way she used to be overwhelmed with patients. She used to call me to assist in registering patients .... this happened regularly.... that is how eventually I became CHW". (3 respondents all women shared similar experience, two from Sengerema, one from Musoma).

\subsection{Economic Gain}

"Sometimes working as CHW pays! Some NGOs pays per-diem during the workshops and even offer us some motivations like bicycles and uniforms" (CHW aged 40 from Sengerema DC)

"Previously we [CHWs] were given some allowances by the government though not every month. But nowadays mmmh! We are not given anything! ...Some CHWs have resigned" (CHW aged 45 from Musoma DC).

"At least those who work with NGOs are paid. But those working in the government are not paid as they expected. Sometimes they are paid; sometimes they stay without any payment for months" (Nurse with 5 years working experience from Musoma DC)

"I can't provide anything to my family if I rely on working as a CHW... we depend on fishing, farming and petty business. .... Government should find a better way of compensating our time and energy! Otherwise it is not worth being a CHW!" (Lamented a female CHW aged 48 from Musoma DC).

She added;

"When CHWs' time is not compensated, the morale to work is diminished eventually they drop from helping us. A number of interviewed health personnel reported" (This was featured in both DCs).

\subsection{Common Identified Interventions and Roles for CHWs}

The current study findings indicated that the involvement of CHWs varied depending on the needs and nature of projects or programs as narrated below;

"We have few CHWs who assist some activities at the Care and Treatment Clinics (CTC), Home-Based Care as well as looking for lost to follow up...we do not have CHW in PMTCT as a separate program" (Narrated a clinical officer with 4 years working experience from Sengerema DC).

"CHWs have been generally involved in $\mathrm{ANC} / \mathrm{RCH}$ and CTC, not been exhaustively involved in PMTCT" (Commented a Clinical Officer from Musoma DC). Similar comment was reported from Sengerema DC.

"We work in ANC/RCH visiting communities for child health follow up, providing immunization, Vitamin A supplementation, de-worming, weighing and recording children in RCH cards" (Said a CHW, woman aged 31 from Musoma DC). This was repeatedly reported across the districts.

\subsection{Views on Challenges Facing $\mathrm{CHWs}$,}

Across the interviews, challenges identified were structural challenges as narrated below,

"This is now my sixth volunteering year. I have been working very hard, no defined roles and responsibilities for us [CHWs] we do various works, yet no incentives have been provided to us...!" (Narrated a male, CHW aged 40 from Sengerema DC). Similar complaints was reported [CHWs] across the interviews.

"We went for a training once, we rarely receive formal backups, mainly we are just learning on job! We need regular backups, close supervisions, monitoring and evaluations. This will help us to perfect what we are doing" (Lamented a female CHW aged 42 from Musoma. Similar complaints were expressed across the interviews.

"When it comes to HIV/AIDS related issues, CHWs are less trusted compared to health personnel. Clients fear that what they share may be spread to the community by CHWs" (commented by a nurse with 12 years working experience from Musoma DC).

\section{Discussion}

This study aimed at assessing the involvement of CHWs in the implementation of PMTCT programs in fishing communities around Lake Victoria in Tanzania. Researchers sought to supplement and provide insights to the existing literature concerning the effectiveness of $\mathrm{CHWs}$ on provision of PMTCT amid inadequacy of HRH as well as in hard to reach communities.

Key findings from this study are categorized into three defining themes; factors that influence community members to work as CHWs, roles played by $\mathrm{CHWs}$, and challenges facing CHWs.

The current study revealed a shortage of HRH services in all health facilities visited, which compelled the engagement of CHWs in the provision of health services. This observation supports $[35,36]$ studies which maintained that deploying CHW's is crucial for effective provision of reliable primary health care services.

The current study identified that motivation for becoming a CHWs is either intrinsic or extrinsic. By being CHWs, majority of KI's expected to receive regular incentives from the facilities, projects or programs. When the extrinsic motives were not realized, the individuals were de-motivated and majorities were less likely to volunteer. Similar CHWs attrition reason have been observed in other studies in Sub Saharan Africa [37] and other poor resource countries [38, 39]. 
In this study, primary tasks of CHWs varied amongst KI's. Most of the tasks involved in included, distribution of deworming drugs and vitamin A supplements (both one-on-one and at public distribution events), and supporting health promotion campaigns.

Further, the current study observed that CHWs were involved much in $\mathrm{MNCH} / \mathrm{ANC}$ and CTC services purposely to curb the challenge of shortage of HRH. Similar CHWs roles have been reported in South Africa [7, 40] in Malawi $[10]$ and Tanzania $[12,13]$.

The current study involving CHWs in PMTCT interventions were not identified to be a common practice in the study area, CHWs were inadequately involved in the implementation of PMTCT services. In most cases, the involvement of CHWs was in $\mathrm{ANC} / \mathrm{RCH}$ and $\mathrm{CTC}$ mainly for curbing the shortage of HRH. On the contrary, in some Sub Saharan countries, the involvement of CHWs has been sought to enhance utilization of PMTCT services. For instance, in their study, Mwai and colleagues reported that CHWs in Malawi, and to a lesser extent in Mozambique, South Africa and Zambia, have been actively mobilizing and referring patients to HIV services including voluntary counseling and testing, Highly Active Antiretroviral Therapy (HAART) initiation, prevention of mother-to-child transmission (PMTCT) and treatment of opportunistic as well as sexually transmitted infections [37]. Comparing to the achievements of CHWs involvement in PMTCT service provision particularly reduced PMTCT prevalence like in the above mentioned countries [37,39], findings from the current study indicated that roles of CHWs in the study area needed to be revisited and CHWs optimally engage in provision of PMTCT services as well.

On the other hand, engaging HIV positive community members in the CHWs teams in providing HIV/ AIDS related services can be fruitful. A systematic review by Ambia and Mandala reports that the involvement of mentor mothers who were HIV-positive with children who were on PMTCT services increased the uptake of PMTCT services in South Africa [41].

The current study found CHWs complaining of not being motivated, similar complaints have been reported from some sub-Saharan countries [37]. Although remuneration to CHWs is currently recommended [5, 38], Bhattacharyya and colleagues agree that maintaining and sustaining the CHWs is hard to ignore the CHWs extrinsic motives but monetary incentives such as wages and salaries should be discouraged. This contradicts with Perry and Zulliger [42] who maintain that lack of appropriate remuneration relative to the assigned workload leads to poor quality of services, loss of motivation, and attrition of CHWs. Other researchers have supported monetary motivation to CHWs. Bhutta and colleagues have recommended that $\mathrm{CHWs}$ who work full time should also be given wages [38]. Other views from Rwanda and India suggest that for maintaining and sustaining the CHWs there is need to enable them to have regular and sustainable personal or group owned sources of income. This can be done by either the government or the development agencies to enable the CHWs to form cooperatives which will enable this strategy $[5,38]$ in so doing the CHWs will concentrate on their duties and responsibilities as long as they are sure of securing income.

The current study revealed that lack of regular back up training and mentorship, lack of supervision, irregular monitoring and evaluation are common challenges in both districts. Similar challenges have been previously reported $[2,21,32]$.

Findings from the current study show that community members had fear of CHWs to expose their private information to other community members. This challenge has been reported elsewhere [43-45]. As it has been recommended, trust between health workers and community members need to be built [46].

\section{Conclusions}

The involvement of CHWs in the provision of HIV/AIDS related services particularly PMTCT in the study areas is inadequate. Deploying CHWs in hard to reach areas such as fishing communities, where $\mathrm{HRH}$ are reported to be inadequate $[15,47]$ is crucial. In order to sustain and maintain the CHWs their fringe benefits should be well stipulated in a national health policy; providing sustainable ways of recruiting, motivating and maintaining CHWs effectively. These provisions should apply to both the government and non-government agencies engaging in healthcare service provision. Future studies ought to focus on areas such as effective approaches for engaging CHWs in hard to reach areas, their performance, efficiency and retention in health care services provision.

\section{Declarations}

\section{Ethical Consideration}

This study was approved by the Kilimanjaro Christian Medical University College Research and Ethics Review Committee; Certificate No1008. Respondents were provided with a written consent for interview and audio recording at enrolment into the study.

\section{Authors' Contributions}

MRM conceptualized the study, collected the data, and did initial analysis and drafting the manuscript. SM and DM participated in review of the manuscript. All authors read and approved the final draft of the manuscript.

\section{Conflict of Interests}

The authors declare that they have no conflict of interests.

\section{Funding}

We thank the Institute of Rural Development Planning (IRDP), Tanzania for sponsoring data collection for this study. 


\section{Availability of Data and Materials}

The dataset generated and analyzed by the current study are not publically available due to confidentiality and protection of the research participants but are available from the corresponding author on reasonable request.

\section{Acknowledgements}

The authors would also like to thank the DMOs and health professionals for Musoma and Sengerema DCs for their support that enabled data collection and the patients for their willingness to participate in this study.

\section{References}

[1] Bangdiwala S, Fonn S, Okoye O, Tollman S. Workforce Resources for Health in Developing Countries. Public Health Reviews. 2010; 32 (1): 296-318.

[2] Byrne A, Hodge A, Jimenez-soto E, Morgan A. What Works? Strategies to Increase Reproductive, Maternal and Child Health in Difficult to Access Mountainous Locations: A Systematic Literature Review. PLoS ONE. 2014; 9 (2): e87683-90.

[3] WHO. Community health workers: what do we know about them? 2014.

[4] Bhattacharyya K, Peter W, LeBan K, Tien M. Community health worker incentives and disincentives: They, How Motivation, Affect. Basic Support for Institutionalizing Child Survival Project (BASICS II). Arlington, Virginia; 2001.

[5] MoH-Rwanda. National Community Health stratetegic Plan July 2013-June 2018. Kigali; 2013.

[6] UNDP. FROM MDGs TO SDGs: GENERAL INTRODUCTION 1. 2015.

[7] Ijumba P. Intervention for improved newborn feeding and survival where HIV is common Perceptions and effects of a community-based package for maternal and newborn care in a South African township. Uppsala University; 2014.

[8] Binagwaho A, Pegurri E, Drobac PC, Mugwaneza P, Stulac $\mathrm{SN}$, Wagner CM, et al. Prevention of mother-to-child transmission of HIV: cost-effectiveness of antiretroviral regimens and feeding options in Rwanda. PloS one. 2013 Jan; 8 (2): e54180-e54180.

[9] MoH Kenya. Minimum Package for RH-HIV Intergration in Kenya. 2012.

[10] Karfakis E, Keehn J. Current Practices to Increase Uptake, Retention and Adherence for Option $\mathrm{B}+$ in Malawi. mothers2mothers Malawi. Lilongwe; 2014.

[11] NAC. Malawi AIDS Response Progress Report 2015. Lilongwe; 2015.

[12] Lema IA, Sando D, Magesa L, Machumi L, Mungure E, Sando MM, et al. Community Health Workers to Improve Antenatal Care and PMTCT Uptake in Dar es Salaam, Tanzania : A Quantitative Performance Evaluation. Journal of Acquired Immune Deficiency Syndromes. 2014; 67: S195201.
[13] Feldhaus I, Silverman M, LeFevre AE, Mpembeni R, Mosha I, Chitama D, et al. Equally able, but unequally accepted: Gender differentials and experiences of community health volunteers promoting maternal, newborn, and child health in Morogoro Region, Tanzania. International journal for equity in health. 2015; 14: 70.

[14] Ahmad K, Zwi AB, Tarantola DJM, Chaudhry TA. SelfPerceived Barriers to Eye Care in a Hard-to-Reach Population: The Karachi Marine Fishing Communities Eye and General Health SurveyBarriers to Eye Care Among Fishing Communities in Karachi. Investigative Ophthalmology \& Visual Science. 2015 Feb 11; 56 (2): 1023-32.

[15] Opio A, Muyonga M, Mulumba N. HIV infection in fishing communities of Lake Victoria Basin of Uganda--a crosssectional sero-behavioral survey. PloS one. 2013 Jan; 8 (8): e70770-80.

[16] Mafigiri R, Matovu JKB, Makumbi FE, Ndyanabo A, Nabukalu D, Sakor M, et al. HIV prevalence and uptake of HIV / AIDS services among youths (15-24 Years) in fishing and neighboring communities of Kasensero, Rakai District, South Western Uganda. BMC public health. 2017; 17: 1-10.

[17] Zafar M, Nisar N, Kadir M, Fatmi Z, Ahmed Z, Shafique K. Knowledge, attitude and practices regarding HIV/AIDS among adult fishermen in coastal areas of Karachi. BMC public health. 2014 Jan; 14 (1): 437.

[18] Kiwanuka N, Mpendo J, Nalutaaya A, Wambuzi M, Nanvubya A, Kitandwe PK, et al. An assessment of fishing communities around Lake Victoria, Uganda, as potential populations for future HIV vaccine efficacy studies: an observational cohort study. BMC public health. 2014; 14.

[19] UAC. Uganda HIV and AIDS Country Progress report 2014. Kampala; 2015.

[20] Kiwanuka N, Ssetaala A, Nalutaaya A, Mpendo J, Wambuzi M, Nanvubya A, et al. High incidence of HIV-1 infection in a general population of fishing communities around Lake Victoria, Uganda. PLoS ONE. 2014; 9.

[21] O'Mara-Eves A, Brunton G, McDaid D, Oliver S, Kavanagh J, Jamal F, et al. Community engagement to reduce inequalities in health: a systematic review, meta-analysis and economic analysis. Public Health Research. 2013; 1 (4): 1-526.

[22] Snow J. The Last Quarter of the Last Mile: Reaching Forgotten Communities in Countries that are Family Planning Success Stories. 2016. p. 2015-7.

[23] NBS. 2012 Population and Housing Census Population Distribution by Adiministrative Area. Dar ES salaam; 2013.

[24] T-MARK. Tanzania Social Marketing Program. Vol. 2013. 2013.

[25] URT. Global Aids Response Country Progress Report. Global AIDS Response Country Progress Report. 2014.

[26] URT-PMoRALG. Mwanza Investment Profile -2013. 2013.

[27] URT. Mara Region Strategic Plan For Accelerated Reduction of Maternal and Newborn Deaths 2013-2016. 2013.

[28] Mwaluko G, Mosha F, Kishamawe C, Mashauri F. The EALP HIV and AIDS Baseline Study in Fishing Communities : Lake Victoria Basin, Tanzania Technical Report African Medical Research Foundation (AMREF). 2011. 
[29] NBS and ICF. Tanzania Demographic and Health Survey 2010. NBS and ICF Macro, editor. National Bureau of Statistics Dar es Salaam, Tanzania ICF Macro Calverton, Maryland, USA. Dar ES salaam; 2011.

[30] URT. The United Republic of Tanzania ministry of health and social welfare human resource for health strategic plan 2008 2013. 2008. 6-91 p.

[31] Afnan-Holmes H, Magoma M, John T, Levira F, Msemo G, Armstrong CE, et al. Tanzania's Countdown to 2015: an analysis of two decades of progress and gaps for reproductive, maternal, newborn, and child health, to inform priorities for post-2015. The Lancet Global Health. 2015 Jul; 3 (7): e396409 .

[32] Nuwagaba-Biribonwoha H, Mayon-White RT, Okong P, Carpenter LM. Challenges faced by health workers in implementing the prevention of mother-to-child HIV transmission (PMTCT) programme in Uganda. Journal of public health (Oxford, England). 2007 Sep; 29 (3): 269-74.

[33] Rooyen H Van, Tulloch O, Mukoma W, Makusha T, Chepuka L, Knight LC, et al. What are the constraints and opportunities for HIVST scale-up in Africa ? Evidence from Kenya, Malawi and South Africa. Journal of the International AIDS Society 2015, 2015; 18: 1-9.

[34] Thomas J, Harden A. Methods for the thematic synthesis of qualitative research in systematic reviews. BMC Medical Research Methodology. 2007; 10: 19.

[35] MoHSW. Health Sector Performance Profile Report 2010 Update, Mainland Tanzania July2009-June 2010. Dar ES salaam; 2010 .

[36] Kwesigabo G, Mwangu AM, Kakoko CD, Mkony AC, Killewo J, Sarah MB, et al. Tanzania' s health system and workforce crisis. Journal of Public Health Policy. 2012; 33 (S1): S35-44.

[37] Mwai G, Mburu G, Torpey K, Frost P, Ford N, Seeley J. Role and outcomes of community health workers in HIV care in sub-Saharan Africa: a systematic review. Journal of the International AIDS Society. 2013; 16: 1-14.

[38] Bhutta ZA, Zohra SL, Pariyo G, Huicho L. Global Experience of Community Health Workers for Delivery of Health Related Millennium Development Goals:A Systematic Review,
Country Case Studies, and Recommendations for Integration into National Health Systems. Geneva; 2009.

[39] Greyling C, Kean S, Perry F, Sengstmann S. Community Prevention of Mother-to-Child Transmission of HIV (cPMTCT) World Vision's Integrated Approach. 2012.

[40] Zarowsky C, Hausler H, Jackson D, Uwimana J. Engagement of non-government organisations and community care workers in collaborative TB/HIV activities including prevention of mother to child transmission in South Africa: Opportunities and challenges. Vol. 12, BMC Health Services Research. 2012. p. 233.

[41] Ambia J, Mandala J. Review article A systematic review of interventions to improve prevention of mother-to-child HIV transmission service delivery and promote retention. Journal of International AIDS Society. 2016; 19 (20309): 1-11.

[42] Perry H, Zulliger R. How Effective are community health workers? An Overview of Current Evidence with Recommendations for Strengthening Community Health Worker Programs to Accelerate Progress in Achieving the Health-related Millennium Development Goals. 2012.

[43] Singh D, Cumming R, Negin J. Acceptability and trust of community health workers offering maternal and newborn health education in rural Uganda. Health Education Research. 2015; 30 (6): 947-58.

[44] Merridy G, Aurene W, Sifiso P, Mntambo N, Christine HM. Trust of community health workers influences the acceptance of community-based maternal and child health services Research methods. African Journal of Primary Health Care and Family Medicene. 2017; 9 (1): 1-8.

[45] Nxumalo N, Goudge J, Manderson L. Community health workers, recipients experiences and constraints to care in South Africa - a pathway to trust. AIDS Care. 2016; 28 sup (4): 61-71.

[46] USAID. Factors impacting the effectiveness of community health worker behavior change a literature review. 2015.

[47] Kwena ZA, Camlin CS, Shisanya CA, Mwanzo I, Bukusi EA. Short-Term Mobility and the Risk of HIV Infection among Married Couples in the Fishing Communities along Lake Victoria, Kenya. PLoS ONE. 2013; 8 (1). 